\title{
Declining Reliance on Medical History and Physical Examination by the Medical Students
}

Received: 7 July 2012 / Accepted: 22 November 2012 / Published online: 28 December 2012

(C) Dr. K C Chaudhuri Foundation 2012

To the Editor: I read with interest the article on the "Decline of the medical history and physical examination" by Akl KF and colleagues [1]. I agree with the authors that the teaching of history taking, physical examination and rational thinking should begin in the medical schools. However many efforts have been made to reform the medical curriculum but these have been unsuccessful in producing changes in the training of medical students. This is because they do not pay any attention to the hidden curriculum which is an important concept in any professional education including medical education. The hidden curriculum consists of what is implicitly taught by example day-to-day, not the explicit teaching of lectures, grand rounds and seminars [2]. Physician role models affect the attitudes, practices and ethics of medical learners and foster professional values in trainees.

I do not think that advances in medical technology alone can be blamed for the decline in clinical skills. Low levels of commitment to teaching, poor teaching skills and repetitive cycles of non-attendance by students and teachers alike are also responsible [3]. Recognition and reform of the hidden curriculum is required to achieve fundamental changes to the culture of under-graduate and post-graduate medical education. However, this is one area in which we the medical fraternity are strangely silent and reluctant to act.

\section{References}

1. Akl KF, Damra HS, Melhem MJ. Decline of the medical history and physical examination. Indian J Pediatr. 2012; 79: 676-7.

2. Gofton W, Regehr G. What we don't know we are teaching: unveiling the hidden curriculum. Clin Orthop Res. 2006; 449: 20-7.

3. Paice E, Heard S, Moss F. How important are role models in making good doctors? BMJ. 2002; 325: $707-10$

\author{
P. Shanbag \\ Department of Pediatrics, ESI-PGIMSR \& MGM Hospital, \\ Dr. S. S. Rao Road, Parel, \\ Mumbai, India \\ P. Shanbag $(\square)$ \\ 801, Yashowan, T.H. Kataria Road, Mahim, \\ Mumbai, India \\ e-mail: pshanbag@gmail.com
}

Author's Reply

To the Editor: We fully agree with comments of Professor Shanbag on our article. Clinical skills, which are the essence of medical practice, should be emphasized on a daily basis at the bedside. As Professor Shanbag mentioned, not only technology is to blame, medical schools need to educate the educators, and make sure that they know how to take history and examine patients. Unfortunately role modeling is also fading away.

\section{Reference}

1. Akl KF, Damra HS, Melhem MJ. Decline of the medical history and physical examination. Indian J Pediatr. 2012; 79: 676-7.

K.F. Akl ( $\square)$, Damra HS, Melhem MJ Department of Pediatrics, Jordan Uiversity Hospital

(JUH), Faculty of Medicine, University of Jordan, P.O.Bx 13046, Amman 11942,

Jordan

e-mail:kachbl@yahoo.com

M.J. Melhem

Intern, Jordan University Hospital (JUH), Faculty of Medicine, University of Jordan,

Amman, Jordan 